\title{
On sums of two $k$ th powers: a mean-square asymptotics over short intervals
}

\author{
by
}

\section{Manfred KüHleitner and Werner Georg Nowak (Wien)}

1. Introduction. For $k \geq 2$ a fixed integer, define the arithmetic function $r_{k}(n)$ as the number of ways to write $n \in \mathbb{N}^{*}$ as a sum of two $k$ th powers of absolute values of integers, i.e.,

$$
r_{k}(n)=\#\left\{\left(u_{1}, u_{2}\right) \in \mathbb{Z}^{2}:\left|u_{1}\right|^{k}+\left|u_{2}\right|^{k}=n\right\} .
$$

To describe its average behaviour, one is interested in asymptotic results about the Dirichlet summatory function

$$
R_{k}(u)=\sum_{1 \leq n \leq u^{k}} r_{k}(n)
$$

where $u$ is a large real variable $\left(^{1}\right)$.

For $k=2$, the classic Gaussian circle problem, a detailed historical exposition can be found in the monograph of Krätzel [10]. The sharpest published results to date $\left({ }^{2}\right)$ read

$$
\begin{gathered}
R_{2}(u)=\pi u^{2}+P_{2}(u), \\
P_{2}(u)=O\left(u^{46 / 73}(\log u)^{315 / 146}\right),
\end{gathered}
$$

and $\left({ }^{3}\right)$

$$
\begin{aligned}
P_{2}(u)=\Omega_{-}\left(u^{1 / 2}(\log u)^{1 / 4}(\log \log u)^{(\log 2) / 4}\right. & \\
\times \exp (-c \sqrt{\log \log \log u})) & (c>0), \\
P_{2}(u)=\Omega_{+}\left(u^{1 / 2} \exp \left(c^{\prime}(\log \log u)^{1 / 4}(\log \log \log u)^{-3 / 4}\right)\right) & \left(c^{\prime}>0\right) .
\end{aligned}
$$

2000 Mathematics Subject Classification: 11P21, 11N37, 11L07.

$\left({ }^{1}\right)$ Note that, in part of the relevant literature, $t=u^{2}$ is used as the basic variable.

$(2)$ Actually, M. Huxley has meanwhile improved further this upper bound, essentially replacing the exponent $46 / 73=0.6301 \ldots$ by $131 / 208=0.6298 \ldots$ The author is indebted to Professor Huxley for sending him a copy of his unpublished manuscript.

$\left({ }^{3}\right)$ We recall that $F_{1}(u)=\Omega_{*}\left(F_{2}(u)\right)$ means that $\lim \sup _{u \rightarrow \infty}\left(* F_{1}(u) / F_{2}(u)\right)>0$ where $*$ is either + or - , and $F_{2}(u)$ is positive for $u$ sufficiently large. 
While (1.2) is due to Huxley [5], [7], (1.3) has been established by Hafner [4], and (1.4) by Corrádi \& Kátai [2]. Most experts conjecture that

$$
\inf \left\{\theta \in \mathbb{R}: P_{2}(u) \ll_{\theta} u^{\theta}\right\}=1 / 2 .
$$

This hypothesis is supported by the mean-square asymptotics

$$
\int_{0}^{T}\left(P_{2}(u)\right)^{2} d u=C_{2} T^{2}+O\left(T(\log T)^{2}\right), \quad C_{2}=\frac{1}{4 \pi^{2}} \sum_{n=1}^{\infty} \frac{\left(r_{2}(n)\right)^{2}}{n^{3 / 2}},
$$

which in this precise form is due to Kátai [8].

The results $(1.3),(1.4),(1.6)$ were obtained by means of the fact that the generating function (Dirichlet series) of $r_{2}(n)$ is the Epstein zeta-function of the quadratic form $u_{1}^{2}+u_{2}^{2}$, which satisfies a well known functional equation and thus opens the possibility of an approach via complex integration.

For the general case $k \geq 3$, quite different methods must be employed. Investigations in this direction have first been undertaken by van der Corput [18] and Krätzel [9]. In Krätzel's textbook [10], an enlightening exposition of the history of the problem including all results until 1988 can be found. It turns out that

$$
R_{k}(u)=\frac{2 \Gamma^{2}(1 / k)}{k \Gamma(2 / k)} u^{2}+B_{k} \Phi_{k}(u) u^{1-1 / k}+P_{k}(u)
$$

where

$$
\begin{aligned}
B_{k} & =2^{3-1 / k} \pi^{-1-1 / k} k^{1 / k} \Gamma\left(1+\frac{1}{k}\right), \\
\Phi_{k}(u) & =\sum_{n=1}^{\infty} n^{-1-1 / k} \sin \left(2 \pi n u-\frac{\pi}{2 k}\right),
\end{aligned}
$$

and the new remainder term $P_{k}(u)$ can essentially be bounded by (1.2), i.e.,

$$
P_{k}(u)=O\left(u^{46 / 73}(\log u)^{315 / 146}\right) .
$$

This was proved by Kuba [11], on the basis of Huxley's method [5], [7].

For lower bounds, it was shown by the second named author [15] that, for any fixed $k \geq 3$,

$$
P_{k}(u)=\Omega_{-}\left(u^{1 / 2}(\log u)^{1 / 4}\right),
$$

and by Kühleitner, Nowak, Schoißengeier \& Wooley [13] that

$$
P_{3}(u)=\Omega_{+}\left(u^{1 / 2}(\log \log u)^{1 / 4}\right) .
$$

The analogy between these results and those for the case $k=2$ might suggest extending the classic conjecture (1.5) to arbitrary $k \geq 2$. In fact, this is true again in mean-square: According to Nowak [14],

$$
\frac{1}{T} \int_{0}^{T}\left(P_{k}(u)\right)^{2} d u \ll T
$$


for any fixed $k \geq 3$ and $T$ large. Kühleitner [12] refined this result, proving an asymptotic formula

$$
\frac{1}{T} \int_{0}^{T}\left(P_{k}(u)\right)^{2} d u=C_{k} T+O\left(T^{1-\varepsilon_{0}(k)}\right),
$$

with explicitly given $\varepsilon_{0}(k)>0$ and

$$
C_{k}:=\frac{4}{\pi^{2}(k-1)} \sum_{\substack{\left(h_{1}, m_{1}, h_{2}, m_{2}\right) \in \mathbb{Z}_{+}^{4} \\\left|\left(h_{1}, m_{1}\right)\right|_{q}=\left|\left(h_{2}, m_{2}\right)\right|_{q}}}\left(h_{1} m_{1} h_{2} m_{2}\right)^{-1+q / 2}\left|\left(h_{1}, m_{1}\right)\right|_{q}^{1-2 q}
$$

Here $q=k /(k-1)$ and $|(h, m)|_{q}=\left(|h|^{q}+|m|^{q}\right)^{1 / q}$ denotes the $q$-norm in $\mathbb{R}^{2}$.

Inspired by a work of Huxley [6] on the lattice point discrepancy of a convex disc, the second named author recently [16] proved a localized form of (1.11), with only a logarithmic loss of accuracy, namely

$$
\int_{T-1 / 2}^{T+1 / 2}\left(P_{k}(u)\right)^{2} d u \ll T(\log T)^{2} .
$$

In view of (1.9), this result seems pretty close to what might be possible. Nevertheless, our aim in the present article is to shed some more light on this short-interval behaviour of this remainder term. It will turn out that the bound in (1.14) (even refined by a factor $\log T$ ) remains valid for an interval up to a length of order $\log T$. In fact, it will be shown that, for any fixed $c_{1}>0$,

$$
\int_{T-c_{1} \log T}^{T+c_{1} \log T}\left(P_{k}(u)\right)^{2} d u \ll T \log T .
$$

Furthermore, we shall see that, as soon as the interval becomes a little longer, we can observe essentially the same asymptotic behaviour as stated in (1.12).

TheOREM. Let $k \geq 3$ be a fixed integer, $T$ a large real variable, and $T \mapsto \Lambda=\Lambda(T)$ an increasing function such that $\Lambda(T) \leq \frac{1}{2} T$ throughout and

$$
\lim _{T \rightarrow \infty} \frac{\log T}{\Lambda(T)}=0 .
$$

Then, as $T \rightarrow \infty$,

$$
\int_{T-\Lambda}^{T+\Lambda}\left(P_{k}(u)\right)^{2} d u \sim 4 C_{k} \Lambda T,
$$

the constant $C_{k}$ being defined in (1.13). 


\section{Two pivotal lemmas}

LEMma 1 (Transition from fractional parts to trigonometric sums according to Vaaler [17]; see also Graham \& Kolesnik [3], p. 116). For arbitrary $w \in \mathbb{R}$ and $H \in \mathbb{N}^{*}$, let

$$
\psi(w)=w-[w]-\frac{1}{2}, \quad \psi_{H}^{*}(w)=-\frac{1}{\pi} \sum_{h=1}^{H} \frac{\sin (2 \pi h w)}{h} \tau\left(\frac{h}{H+1}\right),
$$

where

$$
\tau(\xi)=\pi \xi(1-\xi) \cot (\pi \xi)+\xi \quad \text { for } 0<\xi<1 .
$$

Then

$$
\left|\psi(w)-\psi_{H}^{*}(w)\right| \leq \frac{1}{H+1} \sum_{h=1}^{H}\left(1-\frac{h}{H+1}\right) \cos (2 \pi h w)+\frac{1}{2 H+2} .
$$

Lemma 2. Let $k \geq 3$ be a positive integer, and $q=k /(k-1)$. Then, for $M \rightarrow \infty$,

$$
S(M):=\sum_{\substack{\left(h_{1}, m_{1}, h_{2}, m_{2}\right) \in \mathbb{Z}_{+}^{4} \\\left|\left(h_{1}, m_{1}\right)\right|_{q}=\left|\left(h_{2}, m_{2}\right)\right|_{q} \geq M}}\left(h_{1} h_{2} m_{1} m_{2}\right)^{-1+q / 2}\left|\left(h_{1}, m_{1}\right)\right|_{q}^{1-2 q} \ll M^{-1 / 2} .
$$

Proof. For positive integers $h_{1}, h_{2}, m_{1}, m_{2}$ the condition $\left|\left(h_{1}, m_{1}\right)\right|_{q}=$ $\left|\left(h_{2}, m_{2}\right)\right|_{q}$ is satisfied if and only if either $\left(h_{1}, m_{1}\right)=\left(h_{2}, m_{2}\right)$ or $h_{1}, h_{2}, m_{1}, m_{2}$ all have the same maximal $(k-1)$-free divisor $r$, say, i.e.,

$$
h_{1}=a^{k-1} r, \quad m_{1}=b^{k-1} r, \quad h_{2}=c^{k-1} r, \quad m_{2}=d^{k-1} r,
$$

with $a, b, c, d \in \mathbb{N}^{*}$ satisfying $a^{k}+b^{k}=c^{k}+d^{k}$. This follows from the fact that, by a classic theorem of Besicovitch [1], the $(k-1)$ th roots of distinct $(k-1)$-free positive integers are linearly independent over the rationals. Therefore, the sum in question is

$$
\ll R_{1}(M)+R_{2}(M)
$$

with

$$
\begin{aligned}
R_{1}(M)= & \sum_{\substack{h_{1}=1 \\
m_{1} \gg M}}^{\infty}\left(h_{1} m_{1}\right)^{-2+q}\left(h_{1} m_{1}\right)^{-q+1 / 2}, \\
R_{2}(M)= & \sum_{\substack{a \leq b, c \leq d \\
b^{k-1} r, d^{k-1} r \gg M}}(a b c d)^{(k-1)(-1+q / 2)} \\
& \times r^{-3}\left(\left|\left(a^{k-1}, b^{k-1}\right)\right|_{q}\left|\left(c^{k-1}, d^{k-1}\right)\right|_{q}\right)^{-q+1 / 2},
\end{aligned}
$$

since

$$
\left|\left(h_{1}, m_{1}\right)\right|_{q}=r\left|\left(a^{k-1}, b^{k-1}\right)\right|_{q} \quad \text { and } \quad\left|\left(h_{2}, m_{2}\right)\right|_{q}=r\left|\left(c^{k-1}, d^{k-1}\right)\right|_{q} .
$$


Clearly,

$$
R_{1}(M) \ll \sum_{m_{1} \gg M} m_{1}^{-3 / 2} \ll M^{-1 / 2} .
$$

We estimate the contribution of $R_{2}(M)$ in the cases $k=3,4$, resp. $k \geq 5$ in two different ways. In the first case we use

$$
\frac{1}{\left|\left(u^{k-1}, v^{k-1}\right)\right|_{q}^{q-1 / 2}} \ll(u v)^{-\frac{1}{2}(k-1)(q-1 / 2)},
$$

to conclude that

$$
\begin{aligned}
R_{2}(M) & \ll \sum_{b^{k-1} r, d^{k-1} r \gg M} \sum_{a, c=1}^{\infty}(a b c d)^{(k-1)(-1+q / 2)} r^{-3}(a b c d)^{-\frac{1}{2}(k-1)(q-1 / 2)} \\
& \ll \sum_{r=1}^{\infty} r^{-3}\left(\sum_{b \gg(M / r)^{1 /(k-1)}} b^{-\frac{3}{4}(k-1)}\right)^{2} \ll \sum_{r=1}^{\infty} r^{-3}\left(\frac{M}{r}\right)^{2 /(k-1)-3 / 2} \\
& \ll M^{-1 / 2} .
\end{aligned}
$$

In the case $k \geq 5$ we use the fact that

$$
\sum_{a, c=1}^{\infty}(a c)^{(k-1)(-1+q / 2)} \ll 1,
$$

to infer

$$
\begin{aligned}
R_{2}(M) & \ll \sum_{b^{k-1} r, d^{k-1} r \gg M}(b d)^{(k-1)(-1+q / 2-q+1 / 2)} r^{-3} \\
& \ll \sum_{r=1}^{\infty} r^{-3}\left(\sum_{b \gg(M / r)^{1 /(k-1)}} b^{-k+1 / 2}\right)^{2} \\
& \ll \sum_{r=1}^{\infty} r^{-3}\left(\frac{M}{r}\right)^{(3-2 k) /(k-1)} \ll M^{-7 / 4} .
\end{aligned}
$$

3. Proof of the Theorem. Throughout what follows, let $T$ and $M$ be large real parameters, independent of each other. All constants implied in the symbols $O, \ll$, or $\asymp$ do not depend on $M$ and $T$, but may depend on $k$. Suppose that $u \in[T-\Lambda, T+\Lambda] \subseteq\left[\frac{1}{2} T, \frac{3}{2} T\right]$, thus $u \asymp T$ as $T \rightarrow \infty$.

For any complex-valued function $f: u \mapsto f(u)$ which is square-integrable on $[T-\Lambda, T+\Lambda]$, we shall write

$$
\mathcal{Q}(f)=\mathcal{Q}_{T, \Lambda}(f):=\int_{T-\Lambda}^{T+\Lambda}|f(u)|^{2} d u .
$$


By Cauchy's inequality, for arbitrary $f_{1}, f_{2} \in L^{2}[T-\Lambda, T+\Lambda]$,

$$
\mathcal{Q}\left(f_{1}+f_{2}\right) \leq 2\left(\mathcal{Q}\left(f_{1}\right)+\mathcal{Q}\left(f_{2}\right)\right),
$$

which will be used frequently in what follows.

We start from formulae (3.57), (3.58) (and the asymptotic expansion below) of Krätzel [10], p. 148. In our notation, this reads

$$
P_{k}(u)=-8 \sum_{2^{-1 / k} u<n \leq u} \psi\left(\left(u^{k}-n^{k}\right)^{1 / k}\right)+O(1),
$$

with $\psi(w)=w-[w]-1 / 2$ throughout. We define $q$ by $1 / k+1 / q=1$, i.e., $q=k /(k-1)$, and thus $1<q \leq 3 / 2$. We break up the range of summation into subintervals $\left.\left.\left(^{4}\right) \mathcal{N}_{j}(u)=\right] N_{j}, N_{j+1}\right]$, where $N_{j}=u\left(1+2^{-j q}\right)^{-1 / k}$, $j=0,1, \ldots, J$, with $J$ minimal such that $u-N_{J}<1$ for all $u \in[T-\Lambda, T+\Lambda]$. It is clear that $J \ll \log T$. Furthermore, the length of any $\mathcal{N}_{j}(u)$ is equal to $N_{j+1}-N_{j} \asymp 2^{-j q} T$. By means of Lemma $1, \psi$ will be approximated by $\psi_{H}^{*}$, with $H:=[T]$. Thus overall $P_{k}(u)$ is approximated by

$$
P_{k}^{*}(u):=-8 \sum_{j=0}^{J} \sum_{n \in \mathcal{N}_{j}(u)} \psi_{H}^{*}\left(\left(u^{k}-n^{k}\right)^{1 / k}\right) .
$$

By the definition in Lemma 1 ,

$$
\begin{aligned}
\sum_{n \in \mathcal{N}_{j}(u)} \psi_{H}^{*}\left(\left(u^{k}-n^{k}\right)^{1 / k}\right) & \\
= & -\frac{1}{\pi} \sum_{1 \leq h \leq T} \frac{1}{h} \tau\left(\frac{h}{H+1}\right) \sum_{n \in \mathcal{N}_{j}(u)} \sin \left(2 \pi h\left(u^{k}-n^{k}\right)^{1 / k}\right) .
\end{aligned}
$$

The innermost sum on the right hand side is now subject to a van der Corput transformation ("B-step"). See Kühleitner [12], Lemmas 2 and 3, for details. In particular, we use formula (3.5) from [12] which reads (with $u$ instead of $\sqrt{t}$, and $e(z)=e^{2 \pi i z}$ as usual)

$$
\begin{aligned}
& \sum_{n \in \mathcal{N}_{j}(u)} e\left(-h\left(u^{k}-n^{k}\right)^{1 / k}\right) \\
& =\frac{e(1 / 8)}{\sqrt{k-1}} h u^{1 / 2} \sum_{m \in \mathcal{M}_{j}(h)}^{\prime \prime}(h m)^{-1+q / 2}|(h, m)|_{q}^{-q+1 / 2} e\left(-u|(h, m)|_{q}\right) \\
& \quad+O(j+\log (1+h))
\end{aligned}
$$

$\left({ }^{4}\right)$ The idea of this special choice of subdivision points is that $\frac{d}{d w}\left(\left(u^{k}-w^{k}\right)^{1 / k}\right)$ assumes integer values at $w=N_{j}$. This is useful for the van der Corput transformation of the exponential sums involved. 
where $\mathcal{M}_{j}(h)=\left[2^{j} h, 2^{j+1} h\right],|(h, m)|_{q}=\left(|h|^{q}+|m|^{q}\right)^{1 / q}$ denotes the $q$ norm in $\mathbb{R}^{2}$, and $\sum^{\prime \prime}$ means that the terms corresponding to $m=2^{j} h$ and $m=2^{j+1} h$ get a factor $1 / 2$.

Using the imaginary part of (3.6) in (3.5), we obtain

$$
\begin{aligned}
& \sum_{n \in \mathcal{N}_{j}(u)} \psi_{H}^{*}\left(\left(u^{k}-n^{k}\right)^{1 / k}\right) \\
= & \frac{u^{1 / 2}}{\sqrt{k-1}} \sum_{1 \leq h \leq T} \gamma_{0}(h, T) \sum_{m \in \mathcal{M}_{j}(h)}^{\prime \prime} \frac{(h m)^{-1+q / 2}}{|(h, m)|_{q}^{q-1 / 2}} \sin \left(\pi / 4-2 \pi u|(h, m)|_{q}\right) \\
& +O(\log T)
\end{aligned}
$$

with

$$
\gamma_{0}(h, T):=\frac{1}{\pi} \tau\left(\frac{h}{[T]+1}\right) .
$$

In fact, the main contribution to our mean-square asymptotics will come from a truncation of the double sum here, namely $\left({ }^{5}\right)$

$$
\begin{aligned}
\Sigma_{j}(M, u):= & \frac{u^{1 / 2}}{\sqrt{k-1}} \sum_{1 \leq h \leq T} \gamma_{0}(h, T) \\
& \times \sum_{\substack{m \in \mathcal{M}_{j}(h) \\
|(h, m)|_{q} \leq M}}^{\prime \prime} \frac{(h m)^{-1+q / 2}}{|(h, m)|_{q}^{q-1 / 2}} \sin \left(\pi / 4-2 \pi u|(h, m)|_{q}\right) .
\end{aligned}
$$

What about the errors we commit by these approximations? First of all, evidently,

$$
\begin{aligned}
& \sum_{n \in \mathcal{N}_{j}(u)} \psi_{H}^{*}\left(\left(u^{k}-n^{k}\right)^{1 / k}\right)-\Sigma_{j}(M, u) \\
\ll & T^{1 / 2}\left|\sum_{1 \leq h \leq T} \sum_{m \in \mathcal{M}_{j}(h)}^{\prime \prime} \gamma_{1}(h, m, T) \frac{(h m)^{-1+q / 2}}{|(h, m)|_{q}^{q-1 / 2}} e\left(u|(h, m)|_{q}\right)\right|+\log T
\end{aligned}
$$

with

$$
\gamma_{1}(h, m, T):= \begin{cases}\gamma_{0}(h, T) & \text { if }|(h, m)|_{q}>M \\ 0 & \text { else. }\end{cases}
$$

Furthermore, by Lemma 1,

$$
\begin{aligned}
\sum_{n \in \mathcal{N}_{j}(u)} & \left(\psi\left(\left(u^{k}-n^{k}\right)^{1 / k}\right)-\psi_{H}^{*}\left(\left(u^{k}-n^{k}\right)^{1 / k}\right)\right) \\
& \ll \sum_{1 \leq h \leq T} \frac{1-h /([T]+1)}{[T]+1} \sum_{n \in \mathcal{N}_{j}(u)} \cos \left(2 \pi h\left(u^{k}-n^{k}\right)^{1 / k}\right)+2^{-j q} .
\end{aligned}
$$

$\left({ }^{5}\right)$ Recall that $M$ is another large parameter independent of $T$. 
Applying again (3.6) to the cosine sum here, we see that this is

$$
\ll T^{1 / 2}\left|\sum_{1 \leq h \leq T} \sum_{m \in \mathcal{M}_{j}(h)}^{\prime \prime} \gamma_{2}(h, m, T) \frac{(h m)^{-1+q / 2}}{|(h, m)|_{q}^{q-1 / 2}} e\left(u|(h, m)|_{q}\right)\right|+2^{-j q}
$$

with

$$
\gamma_{2}(h, m, T):=\frac{(1-h /([T]+1)) h}{[T]+1} .
$$

The great similarity of the main parts of the expressions (3.9) and (3.10) enables us to estimate their mean-square by essentially the same calculation. Let

$$
\mathcal{R}_{j}(u):=\sum_{1 \leq h \leq T} \sum_{m \in \mathcal{M}_{j}(h)}^{\prime \prime} \gamma(h, m, T) \frac{(h m)^{-1+q / 2}}{|(h, m)|_{q}^{q-1 / 2}} e\left(u|(h, m)|_{q}\right)
$$

where $\gamma$ is either of $\gamma_{1}, \gamma_{2}$.

We want to bound $\mathcal{Q}\left(\mathcal{R}_{j}\right)$. To this end, we employ an ingenious trick due to Huxley [6] which involves the Fejér kernel

$$
\varphi(w):=\left(\frac{\sin (\pi w)}{\pi w}\right)^{2} .
$$

By Jordan's inequality, $\varphi(w) \geq 4 / \pi^{2}$ for $|w| \leq 1 / 2$, and the Fourier transform has the simple shape

$$
\widehat{\varphi}(y)=\int_{\mathbb{R}} \varphi(w) e(y w) d w=\max (0,1-|y|) .
$$

Therefore,

(3.11) $\mathcal{Q}\left(\mathcal{R}_{j}\right)$

$$
\begin{aligned}
& =2 \Lambda \int_{-1 / 2}^{1 / 2}\left|\mathcal{R}_{j}(T+2 \Lambda w)\right|^{2} d w \leq \frac{\pi^{2}}{2} \Lambda \int_{\mathbb{R}} \varphi(w)\left|\mathcal{R}_{j}(T+2 \Lambda w)\right|^{2} d w \\
& =\frac{\pi^{2}}{2} \Lambda \sum_{1 \leq h_{1}, h_{2} \leq T} \sum_{\substack{m_{1} \in \mathcal{M}_{j}\left(h_{1}\right) \\
m_{2} \in \mathcal{M}_{j}\left(h_{2}\right)}}^{\prime \prime} \frac{\gamma\left(h_{1}, m_{1}, T\right) \gamma\left(h_{2}, m_{2}, T\right)\left(h_{1} m_{1} h_{2} m_{2}\right)^{-1+q / 2}}{\left(\left|\left(h_{1}, m_{1}\right)\right|_{q}\left|\left(h_{2}, m_{2}\right)\right|_{q}\right)^{q-1 / 2}} \\
& \quad \times e\left(-T\left(\left|\left(h_{1}, m_{1}\right)\right|_{q}-\left|\left(h_{2}, m_{2}\right)\right|_{q}\right)\right) \\
& \quad \times \int_{\mathbb{R}} \varphi(w) e\left(-2 \Lambda w\left(\left|\left(h_{1}, m_{1}\right)\right|_{q}-\left|\left(h_{2}, m_{2}\right)\right|_{q}\right)\right) d w \\
& \ll \Lambda \sum_{1 \leq h_{1}, h_{2} \leq T} \sum_{\substack{m_{1} \in \mathcal{M}_{j}\left(h_{1}\right) \\
m_{2} \in \mathcal{M}_{j}\left(h_{2}\right)}}^{\prime \prime} \frac{\gamma\left(h_{1}, m_{1}, T\right) \gamma\left(h_{2}, m_{2}, T\right)\left(h_{1} m_{1} h_{2} m_{2}\right)^{-1+q / 2}}{\left(\left|\left(h_{1}, m_{1}\right)\right|_{q}\left|\left(h_{2}, m_{2}\right)\right|_{q}\right)^{q-1 / 2}}
\end{aligned}
$$$$
\times \max \left(0,1-\left.2 \Lambda||\left(h_{1}, m_{1}\right)\right|_{q}-\left|\left(h_{2}, m_{2}\right)\right|_{q} \mid\right) .
$$ 
We recall that $m \in \mathcal{M}_{j}(h)$ implies that $|(h, m)|_{q} \asymp m \asymp 2^{j} h$. Furthermore, for a term of the last multiple sum to be nonzero it is necessary that $\left.||\left(h_{1}, m_{1}\right)\right|_{q}-\left|\left(h_{2}, m_{2}\right)\right|_{q} \mid<(2 \Lambda)^{-1}$, hence $h_{1} \asymp h_{2}$ and $m_{1} \asymp m_{2}$. Therefore, the last expression in (3.11) is

$$
\begin{aligned}
& \ll \Lambda 2^{-j(q+1)} \sum_{1 \leq h_{1} \leq T} h_{1}^{-3} \sum_{m_{1} \in \mathcal{M}_{j}\left(h_{1}\right)} \gamma\left(h_{1}, m_{1}, T\right) \\
& \times \sum_{\substack{\left(h_{2}, m_{2}\right) \in \mathbb{Z}_{+}^{2} \\
\left|\left(h_{1}, m_{1}\right)\right|_{q}-\left|\left(h_{2}, m_{2}\right)\right|_{q} \mid<(2 \Lambda)^{-1}}} \gamma\left(h_{2}, m_{2}, T\right) .
\end{aligned}
$$

We now have to distinguish if we are dealing with $\gamma_{1}$ or $\gamma_{2}$, recalling the respective definitions: For $\gamma_{1}(h, m, T)$, we know that this is bounded and vanishes for $|(h, m)|_{q} \leq M$. Further, denote by $A_{q}^{*}(U)$ the number of lattice points $\mathbf{v} \in \mathbb{Z}^{2}$ with $|\mathbf{v}|_{q} \leq U$; then it is known that

$$
A_{q}^{*}(U)=\frac{2 \Gamma^{2}(1 / q)}{q \Gamma(2 / q)} U^{2}+O\left(U^{2 / 3}\right)
$$

for any fixed $q$ with $1<q<2$. This asymptotic formula is contained in Theorem 3.6 of Krätzel [10], p. 116. From this it is immediate that, for any fixed $\left(h_{1}, m_{1}\right)$,

$$
\sum_{\substack{\left(h_{2}, m_{2}\right) \in \mathbb{Z}^{2} \\||\left(h_{1}, m_{1}\right)||_{q}-\left|\left(h_{2}, m_{2}\right)\right|_{q} \mid<(2 \Lambda)^{-1}}} 1 \ll \frac{\left|\left(h_{1}, m_{1}\right)\right|_{q}}{\Lambda}+\left|\left(h_{1}, m_{1}\right)\right|_{q}^{2 / 3} .
$$

Thus, for $\gamma=\gamma_{1}$, the expression in (3.12) is

$$
\begin{aligned}
& \ll \Lambda 2^{-j q} \sum_{\substack{1 \leq h_{1} \leq T \\
2^{j} h_{1} \gg M}} h_{1}^{-2}\left(\frac{2^{j} h_{1}}{\Lambda}+\left(2^{j} h_{1}\right)^{2 / 3}\right) \\
& \ll 2^{-j(q-1)} \log T+\Lambda M^{-1 / 6} 2^{-j(q-5 / 6)} \sum_{1 \leq h_{1} \leq T} h_{1}^{-7 / 6} \\
& \ll 2^{-j(q-1)}\left(\log T+\Lambda M^{-1 / 6}\right) .
\end{aligned}
$$

For $\gamma=\gamma_{2}$, we may use that $\gamma_{2}(h, m, T) \ll h T^{-1}$. Thus (3.12) is now, again by $(3.14)$,

$$
\begin{aligned}
(3.16) & \ll \Lambda 2^{-j(q+1)} T^{-2} \sum_{1 \leq h_{1} \leq T} h_{1}^{-1} \sum_{m_{1} \in \mathcal{M}_{j}\left(h_{1}\right)}\left(\frac{\left|\left(h_{1}, m_{1}\right)\right|_{q}}{\Lambda}+\left|\left(h_{1}, m_{1}\right)\right|_{q}^{2 / 3}\right) \\
& \ll \Lambda 2^{-j(q+1)} T^{-2} \sum_{1 \leq h_{1} \leq T}\left(\frac{2^{2 j} h_{1}}{\Lambda}+2^{5 j / 3} h_{1}^{2 / 3}\right) \\
& \ll 2^{-j(q-1)}\left(1+\Lambda T^{-1 / 3}\right) .
\end{aligned}
$$


Let us summarize what we have proved so far: The remainder term in question can be represented as

$$
P_{k}(u)=\sum_{j=0}^{J}\left(-8 \Sigma_{j}(M, u)+\Delta_{j}(M, u)\right),
$$

where $\Sigma_{j}(M, u)$ has been defined in $(3.8)$ and $\Delta_{j}(M, u)$ satisfies (in view of (3.9), (3.10), (3.15), (3.16))

$$
\mathcal{Q}\left(\Delta_{j}(M, u)\right) \ll 2^{-j(q-1)}\left(T \log T+\Lambda T M^{-1 / 6}+\Lambda T^{2 / 3}\right)+\Lambda(\log T)^{2} .
$$

To proceed further, let $\delta$ be a positive constant, less than $\frac{1}{2}(q-1)$ and small compared to $(\log T) / J$. Then, by Cauchy's inequality,

$$
\begin{aligned}
\mathcal{Q}\left(\sum_{j=0}^{J} \Delta_{j}(M, u)\right) & \leq \int_{T-\Lambda}^{T+\Lambda} \sum_{j=0}^{J} 2^{-j \delta} \sum_{j=0}^{J} 2^{j \delta}\left|\Delta_{j}(M, u)\right|^{2} d u \\
& \ll \sum_{j=0}^{J} 2^{j \delta} \mathcal{Q}\left(\Delta_{j}(M, u)\right) \ll T \log T+\Lambda T M^{-1 / 6}+\Lambda T^{2 / 3} .
\end{aligned}
$$

Adding up the main terms $\Sigma_{j}(M, u)$, we arrive at:

Proposition. Uniformly in $T-\Lambda \leq u \leq T+\Lambda$,

$$
P_{k}(u)=\Sigma(M, u)+\Delta(M, u),
$$

with

$$
\mathcal{Q}(\Delta(M, u)) \ll T \log T+\Lambda T M^{-1 / 6}+\Lambda T^{2 / 3}
$$

and

$$
\begin{aligned}
\Sigma(M, u):= & \frac{-8 u^{1 / 2}}{\pi \sqrt{k-1}} \sum_{1 \leq h \leq T} \tau\left(\frac{h}{[T]+1}\right) \\
& \times \sum_{\substack{|(h, m)|_{q} \leq M \\
h \leq m \leq h 2^{J+1}}}^{\prime} \frac{(h m)^{-1+q / 2}}{|(h, m)|_{q}^{q-1 / 2}} \cos \left(\pi / 4+2 \pi u|(h, m)|_{q}\right),
\end{aligned}
$$

where $\sum^{\prime}$ means that the terms corresponding to $m=h$ and $m=h 2^{J+1}$ get a factor $1 / 2$.

Next we infer from the definition in Lemma 1 that $\tau(w)=1+O\left(w^{2}\right)$. Therefore, defining

$$
\begin{aligned}
& \Sigma^{(0)}(M, u) \\
& \quad:=\frac{-8 u^{1 / 2}}{\pi \sqrt{k-1}} \sum_{1 \leq h \leq T} \sum_{\substack{|(h, m)|_{q} \leq M \\
h \leq m \leq h 2^{J+1}}}^{\prime} \frac{(h m)^{-1+q / 2}}{|(h, m)|_{q}^{q-1 / 2}} \cos \left(\pi / 4+2 \pi u|(h, m)|_{q}\right),
\end{aligned}
$$


it is immediate that

$$
\Sigma(M, u)=\Sigma^{(0)}(M, u)+O\left(K_{1}(M) T^{-3 / 2}\right),
$$

where $K_{1}(M), K_{2}(M), \ldots$ will denote appropriate bounds depending on $M$ (but not on $T$ ). If we keep $M$ fixed and make $T$ (and thus $u$ ) large, the summation conditions $h \leq T$ and $m \leq h 2^{J+1}$ ultimately become meaningless, and $\Sigma^{(0)}(M, u)$ becomes equal to

$$
\Sigma^{(1)}(M, u):=\frac{-4 u^{1 / 2}}{\pi \sqrt{k-1}} \sum_{\substack{\left.(h, m)\right|_{q} \leq M \\ h, m \in \mathbb{N}^{*}}} \frac{(h m)^{-1+q / 2}}{|(h, m)|_{q}^{q-1 / 2}} \cos \left(\pi / 4+2 \pi u|(h, m)|_{q}\right) .
$$

We now square out $\left(\Sigma^{(1)}(M, u)\right)^{2}$, using the elementary formula

$$
\cos A \cos B=\frac{1}{2}(\cos (A-B)+\cos (A+B))
$$

and integrate over $u \in[T-\Lambda, T+\Lambda]$. The main contribution comes from the diagonal terms, i.e. those with $\left|\left(h_{1}, m_{1}\right)\right|_{q}=\left|\left(h_{2}, m_{2}\right)\right|_{q}$, and reads altogether

$$
\frac{16}{\pi^{2}(k-1)} \Lambda T \sum_{\substack{\left.\left(h_{1}, m_{1}\right)\right|_{q}=\left|\left(h_{2}, m_{2}\right)\right|_{q} \leq M \\ h_{1}, m_{1}, h_{2}, m_{2} \in \mathbb{N}^{*}}} \frac{\left(h_{1} m_{1} h_{2} m_{2}\right)^{-1+q / 2}}{\left|\left(h_{1}, m_{1}\right)\right|_{q}^{2 q-1}} .
$$

By Lemma 2 and the definition of the constant $C_{k}$ in (1.13), this is equal to

$$
4 \Lambda T\left(C_{k}+O\left(M^{-1 / 2}\right)\right)
$$

All the other terms are pretty small: In fact,

$$
\begin{aligned}
\int_{T-\Lambda}^{T+\Lambda} \sin \left(2 \pi u\left(\left|\left(h_{1}, m_{1}\right)\right|_{q} \pm\left|\left(h_{2}, m_{2}\right)\right|_{q}\right)\right) & u d u \\
& \ll \frac{T}{\left.||\left(h_{1}, m_{1}\right)\right|_{q} \pm\left|\left(h_{2}, m_{2}\right)\right|_{q} \mid},
\end{aligned}
$$

which contributes altogether $\ll K_{2}(M) T$ to $\mathcal{Q}\left(\Sigma^{(1)}(M, u)\right)$. Going back to (3.18) and to the Proposition, and applying Cauchy's inequality one more time, we end up with

$$
\begin{aligned}
\mathcal{Q}\left(P_{k}\right)= & 4 C_{k} \Lambda T+O\left(K_{3}(M) T\right)+O\left(T(\Lambda \log T)^{1 / 2}\right) \\
& +O\left(\Lambda T M^{-1 / 12}\right)+O\left(\Lambda T^{5 / 6}\right) .
\end{aligned}
$$

Therefore, for any fixed $M$, 


$$
\limsup _{T \rightarrow \infty}\left|\frac{1}{\Lambda T} \mathcal{Q}\left(P_{k}\right)-4 C_{k}\right| \ll M^{-1 / 12},
$$

if we recall our condition (1.16). Since $M$ can be chosen arbitrarily large, the proof of our Theorem is thereby complete.

We finally establish (1.15). To this end, it suffices to choose $M=1 / 2$ in the above argument; then all sums over $0<|(h, m)|_{q} \leq M$ are empty, and (3.19) yields what we claimed, since now $\Lambda \asymp \log T$.

\section{References}

[1] A. S. Besicovitch, On the linear independence of fractional powers of integers, J. London Math. Soc. 15 (1940), 3-6.

[2] K. Corrádi and I. Kátai, A comment on K. S. Gangadharan's paper "Two classical lattice point problems", Magyar Tud. Akad. Mat. Fiz. Oszt. Közl. 17 (1967), 89-97 (in Hungarian).

[3] S. W. Graham and G. Kolesnik, Van der Corput's Method of Exponential Sums, Cambridge Univ. Press, Cambridge, 1991.

[4] J. L. Hafner, New omega theorems for two classical lattice point problems, Invent. Math. 63 (1981), 181-186.

[5] M. N. Huxley, Exponential sums and lattice points II, Proc. London Math. Soc. 66 (1993), 279-301.

[6] - The mean lattice point discrepancy, Proc. Edinburgh Math. Soc. 38 (1995), 523-531.

[7] -, Area, Lattice Points, and Exponential Sums, London Math. Soc. Monographs (N.S.) 13, Oxford Univ. Press, 1996.

[8] I. Kátai, The number of lattice points in a circle, Ann. Univ. Sci. Budapest. Eötvös Sect. Math. 8 (1965), 39-60.

[9] E. Krätzel, Bemerkungen zu einem Gitterpunktproblem, Math. Ann. 179 (1969), 90-96.

[10] —, Lattice Points, Deutsch. Verlag Wiss., Berlin, 1988.

[11] G. Kuba, On sums of two k-th powers of numbers in residue classes II, Abh. Math. Sem. Univ. Hamburg 63 (1993), 87-95.

[12] M. Kühleitner, On sums of two kth powers: an asymptotic formula for the mean square of the error term, Acta Arith. 92 (2000), 263-276.

[13] M. Kühleitner, W. G. Nowak, J. Schoißengeier and T. Wooley, On sums of two cubes: An $\Omega_{+}$-estimate for the error term, ibid. 85 (1998), 179-195.

[14] W. G. Nowak, On sums of two $k$-th powers: a mean-square bound for the error term, Analysis 16 (1996), 297-304.

[15] - Sums of two k-th powers: an Omega estimate for the error term, Arch. Math. (Basel) 68 (1997), 27-35.

[16] - On sums of two $k$-th powers: a mean-square bound over short intervals, Funct. Approx. Comment. Math. 28 (2000), 117-125.

[17] J. D. Vaaler, Some extremal problems in Fourier analysis, Bull. Amer. Math. Soc. 12 (1985), 183-216. 
[18] J. G. van der Corput, Over roosterpunkten in het plate vlak, thesis, Groningen, 1919.

Institut für Mathematik und angewandte Statistik

Universität für Bodenkultur

Peter Jordan-Straße 82

A-1190 Wien, Austria

E-mail: kleitner@edv1.boku.ac.at nowak@mail.boku.ac.at

Web: http://www.boku.ac.at/math/nth.html 